\title{
Effect of Diabetes Mellitus and Hypertension on Osmotic Fragility and Hemorheological Factors in Male Wistar Rats
}

\author{
David Ehikhuemen Okonofua ${ }^{1}$, Jerome Ndudi Asiwe ${ }^{1,2, *}$, Kenneth Kelechi Anachuna ${ }^{3}$, \\ Emuesiri Goodies Moke ${ }^{4}$, Kamaldeen Olalekan Sanusi ${ }^{1}$, Ebunoluwa Oluwabusola Adagbada ${ }^{1}$, \\ Mariam Onono Yusuf ${ }^{1}$, Damilola Ifeoluwa Alawode ${ }^{1}$, Adesoji Adedipe Fasanmade ${ }^{1}$ \\ ${ }^{1}$ Cardiorespiratory Research Unit, Department of Physiology, College of Medicine, University of Ibadan, Ibadan, Nigeria. \\ ${ }^{2}$ Department of Physiology, PAMO University of Medical Sciences, Port-Harcourt, Nigeria. \\ ${ }^{3}$ Department of Physiology, Faculty of Basic Medical Sciences, Delta State University, Abraka, Nigeria. \\ ${ }^{4}$ Department of Pharmacology and Therapeutics, Faculty of Basic Medical Sciences, Delta State University, Abraka, Nigeria.
}

Corresponding author*

asiwejerome@yahoo.com; Tel: +2348163727468

Manuscript received: 20 July 2021. Revision accepted: 29 July, 2021. Published: 01 October, 2021.

\begin{abstract}
Diabetes mellitus is a common risk factor for erythrocyte osmotic stress. This study was aimed at exploring the effect of streptozotocin (STZ)-induced diabetes mellitus and salt-induced hypertension on osmotic fragility and hemorheological variables in male Wistar rats. Thirty male rats were grouped into five groups of six animals each as follows: negative control (zero salt in diet); positive control (normal salt diet - 0.3\% salt); high salt diet (8\% salt) (HSD only); STZ induced diabetes and normal salt diet (STZ only); STZ induced diabetes and high salt diet (STZ + HSD). At the end of a 4 weeks period, hematological variables, osmotic fragility, rheology and cardiovascular responses were assessed. There was an increase $(\mathrm{p}<0.05)$ in the mean arterial pressure and heart rate of HSD, STZ and HSD + STZ groups indicating a salt induced hypertension. There was a decrease in the body weight of STZ and HSD +STZ groups. There was significant increase $(\mathrm{p}<0.05)$ in the haematocrit, platelets estimates and fibrinogen concentrations in the experimental groups when compared with the controls. The STZ and STZ + HSD groups showed a reduced clotting time which corresponded to the increased platelet estimates and fibrinogen concentration. The increase in haematocrit, platelet and plasma protein resulted in the increased blood viscosity and a decreased flow rate. The osmotic fragility test was also observed to be increased $(\mathrm{p}<0.05)$ in HSD, STZ only and STZ + HSD groups. Diabetes mellitus and hypertension increase the rate of hemolysis of erythrocyte, as well as increase blood viscosity.
\end{abstract}

Keywords: diabetes mellitus; hemorheology; high salt diet; hypertension; viscosity.

Abbreviations: BP - Blood pressure; DM - Diabetes mellitus; HR - Heart rate; HSD - High salt diet; NaCl - Sodium chloride; RPV Relative plasma viscosity; SBP - Systolic blood pressure; STZ - Streptozotocin; WBV - Whole blood viscosity.

\section{INTRODUCTION}

Diabetes mellitus (DM) is a chronic metabolic disorder in the endocrine system marked by abnormalities in insulin secretion and/or insulin action that leads to the progressive deterioration of glucose tolerance, which causes hyperglycemia (Granner, 2000). In postmodern times, DM has now become a trending public health problem which calls for serious care and concern (Harika et al., 2014). Various medical therapies are current being used in management of diabetes (Patel et al., 2010; Chaudhury et al., 2017; Palanisamy et al., 2018; Okafo et al., 2019). DM is one of the major risk factors for increased osmotic fragility. This is because hyperglycemia causes structural and functional changes in erythrocytes which can result in osmotic stress (Eze et al., 2017).

According to the American Heart Association, high salt consumption is a serious essential/primary hypertension cause and risk factor. Go reported that an average American adult consumes about $5400 \mathrm{mg}$ of sodium per day which is about $130 \%$ increase more than the recommended $2300 \mathrm{mg}$ of sodium per day (Go et al., 2013). This high salt intake per day is a major risk factor for essential hypertension. The rate of erythrocyte osmotic fragility increase in hypertensive subjects is higher than in normotensive subjects (Fasanmade, 1999). High salt intake which induces hypertension will cause an increase in the osmotic fragility of red blood corpuscle (Baskurt and Meiselman, 2003). High salt diets have been greatly linked to be a serious risk factor for cardiovascular diseases relating to high blood pressure, endothelial dysfunction, stroke, ventricular hypertrophy and fibrosis, arterial and ventricular stiffening, myocardial infarction, arrhythmias, and heart failure (Mohan and Campbell, 2009) while diabetes mellitus is a common risk factor for erythrocyte osmotic stress (Eze et al., 2017). This suggests that the 
constituents of blood and its flow properties serve as a link between DM and hypertension.

Hemorheology is the study of the flow properties of blood and its elements (plasma and formed elements, including erythrocytes, white blood cells and platelets) Baskurt et al., 2007). Hemorheological parameters, such as hematocrit, plasma and whole blood viscosity, plasma proteins, erythrocyte deformability and aggregation are basic characteristics of blood flow (Rabai, 2013). There is growing evidence that the flow properties of blood are among the main factors of proper tissue perfusion, and shifts in these properties play significant roles in disease processes (Mohan et al., 2001). The viscosity of blood is directly proportional to the hemoconcentration and inversely proportional to the flow rate. This implies that factors which increase blood constituents and decrease plasma will elevate the blood viscosity which will decrease flow rate and alter tissue perfusion (Chang et al., 2017; Sloop et al., 2020). There are adequate evidences on diabetes that the elevated blood viscosity is a pathogenetic factor of diabetic microangiopathy, altering microcirculation and leading to insufficient tissue nutrition (Grigoleit et al., 1973; Cho et al., 2008). Alterations in tissue perfusion will result in microvascular and macrovascular complications (Cade et al., 2008).

Hence, this study provides an investigation into the rheological properties of blood in diabetic and hypertensive male Wistar rats.

\section{MATERIALS AND METHODS}

\section{Experimental Design}

A total of thirty male Wistar rats (120-150 g) were used for the experiment. They were acclimatized for two weeks prior to commencement of the experiment at the Central Animal House of the College of Medicine, University of Ibadan, Nigeria, and kept under standard laboratory conditions and fed standard rat pellets and water ad libitum. Animal handling was done in accordance to established guidelines by the National Institute of Health for care and use of laboratory animals. Ethical approval was given by the College of Medicine Ethics Committee (UI-ACUREC/19/0140).

The rats were grouped into five groups of six animals each as follows: negative control (zero salt in diet); positive control (normal salt diet $-0.3 \%$ salt); high salt diet ( $8 \%$ salt) (HSD only); STZ induced diabetes and normal salt diet (STZ only); STZ induced diabetes and high salt diet (STZ + HSD)

\section{Preparation of Feed Rations}

Three different rations were used for the study. The high salt diet was prepared by mixing $8 \mathrm{~g}$ of table salt with 92 $\mathrm{g}$ of standard rat chow (Asiwe et al., 2020). HSD only and STZ +HSD groups were given this ration and water ad libitum. The normal salt diet was prepared by mixing
$0.30 \mathrm{~g}$ of table salt with $99.70 \mathrm{~g}$ of standard rat chow which was given to the positive control group and water ad libitum. While the zero-salt diet was given to the negative control group.

\section{Induction of Diabetes Mellitus}

Diabetes was induced by single intraperitoneal injection of $60 \mathrm{mg} / \mathrm{kg}$ body weight dose of streptozotocin (STZ) dissolved in freshly prepared $0.1 \mathrm{M}$ cold citrate buffer of $\mathrm{pH} 4.5$ into the animals according to the STZ-induced hyperglycemia in rats' model (Eze et al., 2017; Asiwe et al., 2020; Akbarzadeh et al., 2007). The experimental animals were fasted overnight (18 hours) prior to diabetes induction while allowed access to drinking water. Seventy- two hours after streptozotocin injection, a drop of blood was drawn from tail vein of the rats to measure their blood glucose level. Animals having fasting blood glucose levels $\geq 200 \mathrm{mg} / \mathrm{dL}$ were considered diabetic and used in the study.

\section{Measurement of Blood Glucose Level}

In order to ascertain the diabetic state of the animals, the fasting blood sugar level was measured every week after being fasted overnight using the ACCU-CHEK Active glucometer (Model GB06140695). The results obtained in $\mathrm{mmol} / \mathrm{L}$ were converted to $\mathrm{mg} / \mathrm{dL}$ by multiplying with 18 (conversion factor). Blood samples were collected from tail artery of the rats for evaluation of the blood glucose. Body weights of animals were also measured weekly in grams to estimate the effect of the induced diabetes and high salt diet on body composition. The weight was measured with a standard weighing scale.

\section{Blood Pressure and Heart Rate}

Blood Pressure and Heart Rate were done at the Small Animal Ward of the Veterinary Clinic University of Ibadan, Ibadan, Nigeria. Systolic Blood Pressure (SBP) was measured indirectly in a conscious and slightly restrained rat using the tail cuff plethysmography method (Kent Scientific, USA). Heart Rate (HR) tracings were observed and recorded as obtained during blood pressure (BP) measurement. For these measurements, rats were conditioned to the restraint (cone) and the warming chamber for about 20 minutes before the measurement. SBP and HR measurements were performed in a very quiet environment to avoid sound interference by the same investigator. There were two sensors to measure BP and Vascular Peripheral Resistance. After stabilization in the chamber, an acclimatization run was performed for 5 cycles which was immediately followed by the typical run involving 10 repetitions of the automated inflation-deflation cycle.

\section{Examination of Blood Samples}

Blood samples were collected by ocular puncture at the end of the fourth week and stored in heparinized bottles which were used to consider effect of high salt diet and 
diabetes mellitus on hematology, osmotic fragility and rheology. Four animals were used for each group for analysis $(n=4)$.

\section{Rheological Analysis}

Whole blood viscosity and relative plasma viscosity were estimated using the method described by Reid and Ugwu (1987), and the flow rate was calculated. Plasma fibrinogen concentration was estimated by clot weight method of Ingram (1952). Haematocrit was estimated using microhaematocrit reader.

\section{Statistical Analysis}

Data were analyzed with GraphPad Prism version 7.0 (GraphPad Software, San Diego, CA) and expressed as means \pm SEM (standard error of mean). One-way ANOVA was used for comparisons, followed by post hoc Newman-Keuls Multiple Comparison test. P $<0.05$ was considered statistically significant.

\section{RESULTS AND DISCUSSION}

\section{Osmotic Fragility}

Figure 1 shows the rate of erythrocyte osmotic fragility $(\%)$. The percentage erythrocyte osmotic fragility decreased significantly with an increase in sodium chloride $(\mathrm{NaCl})$ concentration. There was complete $(100 \%)$ haemolysis at $0.0 \%$ of $\mathrm{NaCl}$. And there were no significant changes in erythrocyte osmotic fragility when observed at $0.0 \%$ and $0.1 \%$ of $\mathrm{NaCl}$ concentration in all control and experimental groups when compared. However, significant $(\mathrm{P}<0.05)$ changes in percentage erythrocyte fragility were recorded at $0.3 \%, 0.5 \%, 0.7 \%$ and $0.9 \%$ of $\mathrm{NaCl}$ concentrations, when the experimental groups were compared with the control groups. $*_{\mathrm{a}}, *_{\mathrm{b}},{ }^{*} \mathrm{c}$, and $*_{\mathrm{d}} \mathrm{p}<0.05$ at $0.3 \%, 0.5 \%, 0.7 \%$ and $0.9 \%$ of $\mathrm{NaCl}$ concentrations.

\section{Platelet Counts}

Figure 2 shows the platelets estimates $\left(\mathrm{mm}^{3}\right)$ in diabetic and hypertensive male Wistar rats. There was significant increase when High salt diet + STZ groups were compared with the controls. $p<0.05$ is significant when compared with negative control and positive control groups.

\section{Fibrinogen Estimates}

Figure 3 shows the fibrinogen estimates $\left(\mathrm{mm}^{3}\right)$ in diabetic and hypertensive male wistar rats. There was significant increase when other groups were compared with the controls. *p $<0.05$ is significant when compared with negative control and positive control groups.

\section{Hematocrit}

Figure 4 shows the hematocrit (\%) in diabetic and hypertensive male wistar rats. There was significant increase when other groups were compared with the controls. $* \mathrm{p}<0.05$ is significant when compared with negative control and positive control groups.

\section{Whole Blood Viscosity}

Figure 5 shows the whole blood viscosity (WBV) (mPa.s) in diabetic and hypertensive male wistar rats. From the values expressed there was significant increase between the negative control group and positive control group. There was significant increase when other groups were compared with the controls. **p $<0.05$ is significant when the negative control group was compared with the positive control group; ${ }^{*} \mathrm{p}<0.05$ is significant when compared with negative control and positive control groups.

\section{Relative Plasma Viscosity}

Figure 6 shows the Relative Plasma Viscosity (RPV) (mPa.s) in diabetic and hypertensive male wistar rats. From the values expressed there was significant increase between the negative control group and positive control group. There was significant increase when other groups were compared with the controls. **p $<0.05$ is significant when the negative control group was compared with the positive control group; ${ }^{*} \mathrm{p}<0.05$ is significant when compared with negative control and positive control groups.

\section{Plasma Flow Rate}

Figure 7 shows the plasma flow rate $(\mathrm{cm} / \mathrm{sec})$ in diabetic and hypertensive male wistar rats. There was significant increase between the negative control and positive control groups. There was significant increase when other groups were compared with the controls. $* *$ p $<$ 0.05 is significant when the negative control group was compared with the positive control group; ${ }^{*} \mathrm{p}<0.05$ is significant when compared with negative control and positive control groups.

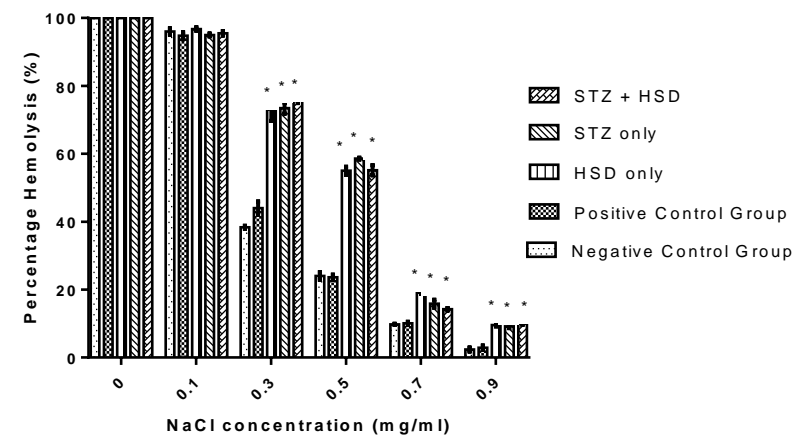

Figure 1. Rate of erythrocyte osmotic fragility (\%) in diabetic and hypertensive male Wistar rats. 


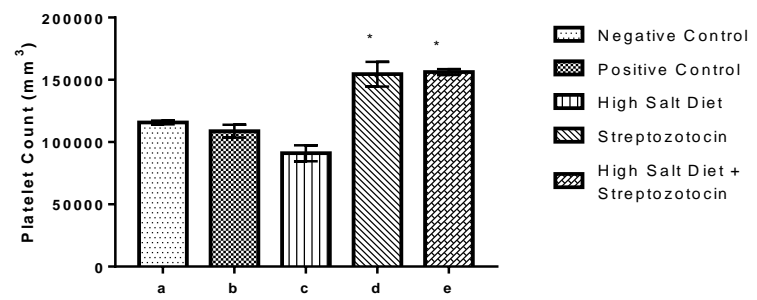

Figure 2. Platelet count $\left(\mathrm{mm}^{3}\right)$ in diabetic and hypertensive male Wistar rats.

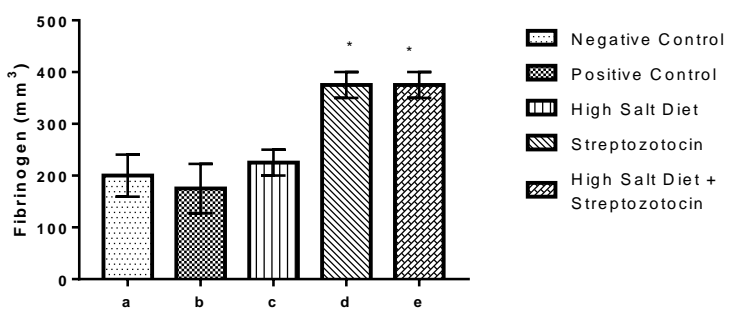

Figure 3. Fibrinogen estimates $\left(\mathrm{mm}^{3}\right)$ in diabetic and hypertensive male Wistar rats.

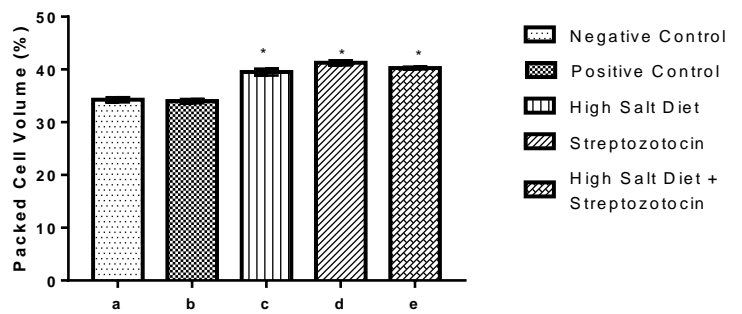

Figure 4. Hematocrit (\%) in diabetic and hypertensive male Wistar rats.

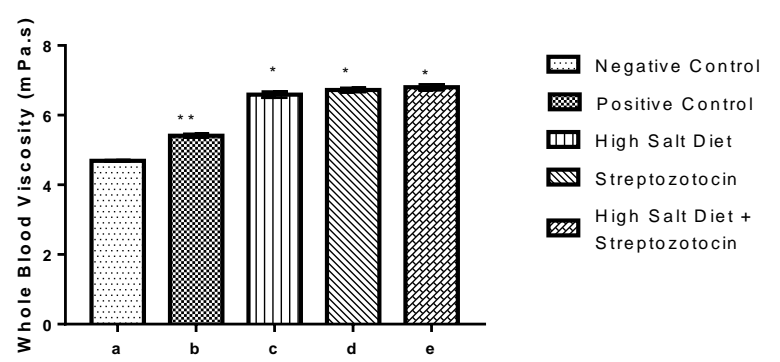

Figure 5. Whole Blood Viscosity (mPa.s) in diabetic and hypertensive male Wistar rats.

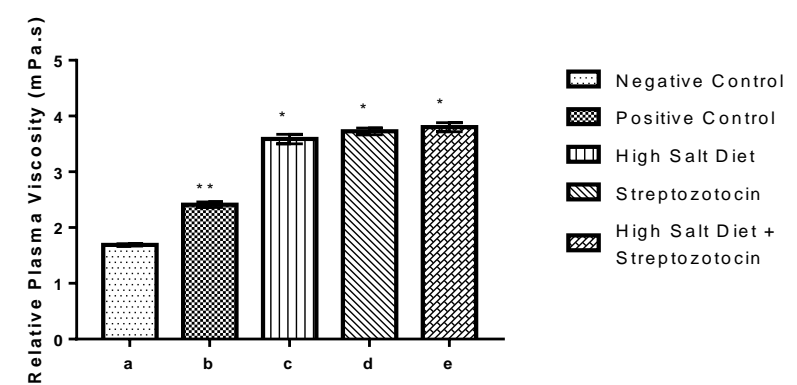

Figure 6. Relative Plasma Viscosity (mPa.s) in diabetic and hypertensive male Wistar rats.

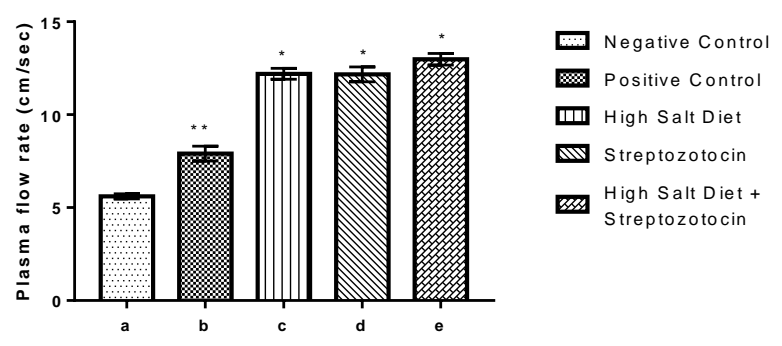

Figure 7. Plasma flow rate $(\mathrm{cm} / \mathrm{sec})$ in diabetic and hypertensive male Wistar rats.

In this study, the percentage erythrocyte osmotic fragility decreased significantly with increasing $\mathrm{NaCl}$ concentration. There was complete $(100 \%)$ hemolysis at $0.0 \%$ and $0.1 \%$ of $\mathrm{NaCl}$. No significant changes in erythrocyte osmotic fragility were observed at $0.0 \%$ and $0.1 \%$ of $\mathrm{NaCl}$ (distilled water) in both controls and experimental groups when compared. However, the results of the osmotic fragility showed a significant increase in the rate of erythrocyte osmotic fragility at $0.3 \%, 0.5 \%, 0.7 \%$ and $0.9 \%$ of $\mathrm{NaCl}$ concentrations in HSD only, STZ only and HSD +STZ groups, when compared with the control groups. This finding was in agreement with the works of other researchers (Shin et al., 2007; Megha and Sehyun, 2009; Eze et al, 2017) who showed that erythrocyte osmotic fragility was significantly increased in streptozotocin-induced diabetic animals. The increased rate of erythrocyte osmotic fragility in hypertensive subjects has also been reported by Fasanmade (1999). The implication of this finding suggests that STZ induced diabetes can alter the integrity of the red blood cell (RBC) membrane.

Erythrocyte osmotic fragility test gives an in-vitro measure of the tensile strength of erythrocyte membrane and it is an indirect method of evaluating lipid peroxidation in animals (Akande et al., 2014). It is also used to measure the tensile strength of erythrocytes and its ability to resist alterations in osmotic gradients and it has been discovered to be increased during oxidative stress (Aldrich et al., 2006; Adenkola et al., 2010). Hence, this suggests that the greater the erythrocyte osmotic fragility, the weaker the tensile strength of erythrocyte membrane (Ogundeji et al, 2013) and a resultant hemolysis of erythrocyte is observed. The mechanism for increased fragility of erythrocytes have been reported to be due to increased glycosylation of the erythrocyte membrane protein or/and alteration of the $\mathrm{Na}+/ \mathrm{K}+$ ATPase on the erythrocyte membrane (Arun, 2013). The viability of an erythrocyte depends greatly on the maintenance of its membrane. Studies have shown that DM and hypertension can cause an increase in lipoperoxidative changes in the membranes of erythrocytes (Qujeq et al., 2005; Ahmed et al., 2006; Gauri and Vijaya, 2008) which account for the increase in the rate of osmotic fragility.

Hemorheology is the study of the flow properties of blood and its elements such as plasma with its dissolved 
components and formed elements, which include erythrocytes, leucocytes and platelets (Baskurt et al., 2007). The flow properties of blood are among the main determinants of proper tissue perfusion, and alterations in these properties play vital roles in disease processes (Lowe et al., 1980). In this study, hemorheological factors were measured with focus on blood viscosity and flow rate. The viscosity of blood is determined by the hematocrit (Rabai, 2013; Cho et al., 2008), increase in plasma viscosity which is determined by plasma proteins (fibrinogen and globulin) and water (Mohan et al., 2001). From this study, there was a significant increase in the blood viscosity and plasma viscosity in the HSD only, STZ only and STZ+HSD groups when compared with the control groups. The observed increase in viscosity could be due to the significant increase in hematocrit, fibrinogen, and platelet estimates as recorded in STZ only and STZ+HSD groups. This finding was in accordance with the works of Reid and Memeh, and others (Reid and Memeh, 1988; Khan et al., 2005; Okomafe et al., 2017). Increased blood viscosity and the onset of diabetic angiopathy have been related to abnormal hematocrit, plasma viscosity, fibrinogen concentration, erythrocyte deformability and rouleaux formation (Winberger and Baskurt, 2007). The increase in hematocrit in the experimental groups appears to have contradicted the increased rate of erythrocyte osmotic fragility as reported earlier in this study. A possible mechanism to support this finding is linked to hemoconcentration. As the osmolarity of the blood increases due to increased glucose level, the capillary permeability increases, resulting in a decrease in plasma water and thus increasing hematocrit and subsequently the blood viscosity (Meiselman et al., 1967; Rizvi and Zaid, 2001). However, HSD only group had a significant increase in hematocrit only with a decrease in fibrinogen and platelet estimates when compared with other experimental groups. This increase in hematocrit could be the major reason behind the increase in blood viscosity (MacRury et al., 1988). Another interesting finding in this study was the significant increase in blood and plasma viscosity when the positive control group with normal salt intake was compared with the salt deficient negative control group. The possible mechanism underlying this claim is yet to be fully understood.

The flow rate of blood is determined by three main factors - vessel diameter, perfusion pressure and blood viscosity (Lowe et al., 1980). Although vessel diameter has been primarily linked with blood flow rate, in pathological conditions, the contributions of perfusion pressure and viscosity becomes greatly enhanced. It has been reported from studies that the viscosity of blood is directly proportional to the hemoconcentration and inversely proportional to the flow rate (Sloop et al., 2020). This implies that factors which increase blood constituents and decrease plasma water will elevate the viscosity of blood which will decrease flow rate and alter tissue perfusion (Chang et al., 2017; Sloop et al., 2020). From this study, it was also observed that there was a corresponding significant decrease in the flow rate of plasma across the experimental groups when compared with the control groups. Hence, the increase in blood viscosity could be a factor for the decreased flow rate of plasma. The alterations in the blood flow patterns in DM is produced by a combination of reduced erythrocyte deformability and increased erythrocyte aggregation due to variations in the plasma protein (McMillian et al., 1978). The changes in plasma proteins are linked with the development of glucose intolerance (McMillian, 1983). A decrease in flow rate can possibly result in circulatory insufficiency marked with poor tissue perfusion which in a long term can lead to the development of vascular complications as commonly observed in subjects with DM (Lowe et al., 1980). There was a significant decrease in plasma flow rate when the positive control group with normal salt intake (with higher blood and plasma viscosity) was compared with the salt deficient negative control group. This also verified the inverse relationship between blood viscosity and plasma flow rate.

\section{CONCLUSION}

From the findings of this study, it can be stated that diabetes mellitus and hypertension increase the rate of hemolysis of erythrocyte. There is also an increase in the hematocrit which significantly increases the blood viscosity alongside with plasma protein and decreased plasma water, thus, decreasing plasma flow rate. This could possibly result in circulatory insufficiency as well as a poor tissue perfusion which will lead to vascular complications.

Conflict of Interest: The authors declare that there are no conflicts of interest concerning the publication of this article.

\section{REFERENCES}

Adenkola AY, Ayo JO, Sackey AKB, Adelaiye AB (2010). Erythrocyte Osmotic Fragility of Pigs Administerd Ascorbic Acid and Transported by Road for Short Term Duration during the Harmattan Season. Afr J Biotechnol 9: 226-233.

Ahmed FN, Naqvi FN, Shafiq F (2006). Lipid Peroxidation and Serum Antioxidant Enzymes in Patients with Type 2 Diabetes Mellitus. Ann N Y Acad Sci 1084: 481-489.

Akande MG, Aliu YO, Ambali SF, Ayo JO (2014). Protective Effect of Taurine in Chlorpyrifos and Lead-Induced Haematological Alterations in Wistar Rats. Toxicol Environ Chem 96: 171-182.

Akbarzadeh A, Norouzian D, Mehrabi MR, Jamshidi Sh, Farhangi A, Verdi AA, Mofidian SM, Rad BL (2007). Induction of diabetes by Streptozotocin in rats. Indian J Clin Biochem 2(2): 60-64. 
Aldrich KJ, Saunders DK, Sievert LM, Sievert G (2006). Comparison of Erythrocyte Osmotic Fragility among Amphibians, Reptiles, Birds and Mammals. Trans Kans Acad Sci 109: 149-158.

Arun PM (2013). Erythrocyte Fragility Increases with the Duration of Diabetes in Indian Population. IJBAMS 3: 172177.

Asiwe JN, Anachuna KK, Moke EG, Sanusi KO, Okonofua DE, Omeru O, Fasanmade AA (2020). High dietary salt intake alleviates fasting blood glucose in streptozotocin-induced diabetic male Wistar rats. Thai J Pharm Sci 45(3): 172-177.

Baskurt OK, Hardeman MR, Rampling MW, Meiselman HJ (2007). Handbook of hemorheology and hemodynamics, IOS Press, Amsterdam.

Baskurt OK, Meiselman HJ (2003). Blood Rheology and Hemodynamics. Semin Thromb Hemost 29(5): 435-450.

Cade WT (2008). Diabetes-related microvascular and macrovascular diseases in the physical therapy setting. Phys Ther 88: 1322-1335.

Chang HY, Li X, Karniadakis GE (2017). Modeling of Biomechanics and Biorheology of Red Blood Cells in Type 2 Diabetes Mellitus. Biophys J 113(2): 481-490.

Chaudhury A, Duvoor C, Reddy Dendi VS, Kraleti S, Chada A, Ravilla R, Marco A, Shekhawat NS, Montales MT, Kuriakose K, Sasapu A, Beebe A, Patil N, Musham CK, Lohani GP, Mirza W (2017). Clinical Review of Antidiabetic Drugs: Implications for Type 2 Diabetes Mellitus Management. Front Endocrinol (Lausanne) 8: 6.

Cho YI, Mooney MP, Cho DJ (2008). Hemorheological disorders in diabetes mellitus. J. Diabetes Sci. Technol 2: 1130-1138.

Eze ED, Tanko Y, Abubakar A, Sulaiman SO, Rabiu KM, Mohammed A (2017). Lycopene Ameliorates DiabeticInduced Changes in Erythrocyte Osmotic Fragility and Lipid Peroxidation in Wistar Rats. J Diabetes Mellitus 7: 71-85.

Fasanmade AA (1999). Erythrocyte Osmotic Fragility in Hypertension and during Diuretic Therapy. West Afr J Med 15(3): 183-186.

Gauri SV, Vijaya AH (2008). RBC Membrane Composition in Insulin Dependent Diabetes Mellitus in Context of Oxidative Stress. Indian J Clin Biochem 23: 223-226.

Go AS, Mozaffarian D, Roger VL, Benjamin EJ, Berry JD, Borden WB, et al (2013). American Heart Association Statistics Committee and Stroke Statistics Subcommittee. Heart disease and stroke statistics--2013 update: a report from the American Heart Association. Circulation. 127(1): e6-e245.

Granner DK (2000). Hormones of the Pancreas and Gastrointestinal Tract. In: Meyes PA, editor. Harpers Biochemistry, McGraw Hill, New York, 610-626.

Grigoleit HG, Lehrach F, Muller R (1973). Diabetic angiopathy and blood viscosity. Acta Diabet Lat 10: 1311-1324.

Harika PK, Asha LP, Pradnya S, Ayesha J, Samatha P, Mani RK (2014). Comparative Study of Erythrocyte Fragility in Diabetes Mellitus and Nondiabetes Mellitus. IJMRHS 4(1): 183-185.

Ingram GIC (1952). The determination of plasma fibrinogen by the clot-weight method. Biochem J 51(5): 583-585.

Khan TM, Marwat MA, Khan P, Farman-ullah W, Amir R (2005). Plasma fibrinogen level in diabetics with complications - A prospective study. Gomal J Med Sci 3(2): 48-50

Lowe GD, Lowe JM, Drummond MM, Reith S, Belch JJ, Kesson CM, Wylie A, Foulds WS, Forbes CD, MacCuish AC,
Manderson WG. (1980). Blood viscosity in young male diabetics with and without retinopathy. DiabetoIogia 18(5): 359-363.

MacRury SM, Small M, MacCuish AC, Lowe DC (1988). Association of Hypertension with Blood Viscosity in Diabetes. Diabet Med 5: 830-834.

McMillan D (1983). The effect of diabetes mellitus on blood flow properties. Diabetes 7: 85-90.

McMillan DE, Utterback NG, Puma JL (1978). Reduced erythrocyte deformability in diabetes. Diabetes 27: 895-901.

Megha S, Sehyun S (2009). Changes in Erythrocyte Aggregation and Deformability in Diabetes Mellitus: A Brief Review. Indian J Exp Biol 47: 7-15.

Meiselman HJ, Merrill EW, Gilliland ER, Pelletier GA, Salzman EW (1967). Influence of plasma osmolarity on the rheology of human blood. J Appl Physiol 22(4): 772-781.

Mohan A, Srinivasan V, Deepa R, Mohan V (2001). Lipoprotein(a): role in diabetes and its vascular complications. JAPI 49: 1100-1105.

Mohan S, Campbell NR (2009). Salt and high blood pressure. Clin Sci 117(1): 1-11.

Ogundeji T, Ayo JO, Aluwong T, Mohammed A (2013). Behavioural and haematological studies on effects of lycopene in wistar rats subjected to psychologicalstress. J Neurosci Behav Health 5: 30-35.

Okafo SE, Moke EG, Obi CS (2019). Formulation and evaluation of anti-diabetic tablets containing aqueous extract of Moringa oleifera seeds. JOPHAS 16(5): 3167-3176.

Okomafe NE, Oluranti OI, Fasanmade AA (2017). Effect of sleep deprivation on hemorheological properties in alloxan induced diabetic rats. EJMR 6(2): 16-21.

Palanisamy S, Yien ELH, Shi LW, Si LY, Qi SH, Ling LSC, Lun TW, Chen YN (2018). Systematic Review of Efficacy and Safety of Newer Antidiabetic Drugs Approved from 2013 to 2017 in Controlling HbA1c in Diabetes Patients. Pharmacy (Basel) 6(3): 57.

Patel DK, Prasad SK, Kumar R, Hemalatha S (2012). An overview on antidiabetic medicinal plants having insulin mimetic property. Asian Pac J Trop Biomed 2(4): 320-30.

Qujeq D, Habibinudeh M, Daylmkatoli H, Rezvani T (2005). Malondialdehyde and Carbonyl Contents in the Erythrocytes of Streptozotocin-Induced Diabetic rats. Int J Diabetes Metab 13: 96-98.

Rabai M (2012). In vitro hemorheological studies focusing on erythrocyte deformability and aggregation [Ph.D. thesis], University of Pecs, Hungary.

Reid HL, Memeh CU (1988). Plasma and serum viscosity in diabetes mellitus. Nig J Physiol Sci 4: 71-72

Reid HL, Ugwu AC (1987). A simple technique for rapid determination of plasma viscosity. Nig J Physiol Sci 3: 45-48

Rizvi SI, Zaid MA (2001). Intracellular reduced glutathione content in normal and type 2 diabetic erythrocytes: effect of insulin and (-) epicatechin. J Physiol Pharmacol 52(3): 483488

Shin S, Ku Y, Babu N, Singh M (2007). Erythrocyte Deformability and Its Variation in Diabetes Mellitus. Indian J Exp Biol 45: 121-128.

Sloop GD, De Mast Q, Pop G, Weidman JJ, St Cyr JA (2020). The Role of Blood Viscosity in Infectious Diseases. Cureus 12(2): e7090. 
Winberger U, Baskurt OK (2007). Comparative hemorheology. Baskurt OK, Hardeman MR, Rampling MW, Meiselman HJ, editors. Handbook of hemorheology and hemodynamics, IOS Press, Amsterdam, 267-285. 
THIS PAGE INTENTIONALLY LEFT BLANK 